\title{
Recollection-Related Increases in Functional Connectivity Predict Individual Differences in Memory Accuracy
}

\author{
(D)Danielle R. King, Marianne de Chastelaine, Rachael L. Elward, Tracy H. Wang, and Michael D. Rugg \\ Center for Vital Longevity and School of Behavioral and Brain Sciences, University of Texas at Dallas, Dallas, Texas 75235
}

Recollection involves retrieving specific contextual details about a prior event. Functional neuroimaging studies have identified several brain regions that are consistently more active during successful versus failed recollection-the "core recollection network." In the present study, we investigated whether these regions demonstrate recollection-related increases not only in activity but also in functional connectivity in healthy human adults. We used fMRI to compare time-series correlations during successful versus unsuccessful recollection in three separate experiments, each using a different operational definition of recollection. Across experiments, a broadly distributed set of regions consistently exhibited recollection-related increases in connectivity with different members of the core recollection network. Regions that demonstrated this effect included both recollection-sensitive regions and areas where activity did not vary as a function of recollection success. In addition, in all three experiments the magnitude of connectivity increases correlated across individuals with recollection accuracy in areas diffusely distributed throughout the brain. These findings suggest that enhanced functional interactions between distributed brain regions are a signature of successful recollection. In addition, these findings demonstrate that examining dynamic modulations in functional connectivity during episodic retrieval will likely provide valuable insight into neural mechanisms underlying individual differences in memory performance.

Key words: angular gyrus; episodic memory; fMRI; functional connectivity; hippocampus; recollection

\section{Introduction}

Recollection refers to the processes that support retrieval of qualitative information about a prior event (Mandler, 1980; Yonelinas, 2002). Recollection can be contrasted with familiarity; recognition without retrieval of qualitative details. Findings from functional neuroimaging studies have demonstrated that a set of brain regions are more active during recollection than familiarity-based recognition (Spaniol et al., 2009; Kim, 2010; Rugg and Vilberg, 2013). Regions belonging to this "core recollection network" include left angular gyrus, posterior cingulate/retrosplenial cortex, medial prefrontal cortex, hippocampus, and parahippocampal cortex.

Although this "modular" approach to characterizing fMRI findings has advanced our understanding of the neural substrates of recollection, the benefits of adopting a complementary approach have become increasingly evident. This approach involves investigating interactions between different brain regions. One method of assessing "functional connectivity" involves analyzing time-dependent fluctuations in blood oxygen level-dependent (BOLD) signal time courses acquired over a sustained period (Greicius et al., 2003; Fox et al., 2005; Wig et al., 2011). If the time

Received Aug. 4, 2014; revised Dec. 1, 2014; accepted Dec. 5, 2014.

Author contributions: M.d.C., R.L.E., T.H.W., and M.D.R. designed research; M.d.C., R.L.E., T.H.W., and M.D.R. performed research; D.R.K., M.d.C., R.L.E., T.H.W., and M.D.R. analyzed data; D.R.K. and M.D.R. wrote the paper.

This research was supported by NIMH Grant 5R01MH072966 and NIA Grant 1R01AG039103.

The authors declare no competing financial interests.

Correspondence should be addressed to Dr Danielle R. King, 1600 Viceroy Drive, Suite 800, Dallas, TX 75235. E-mail:dking@utdallas.edu.

DOI:10.1523/JNEUROSCI.3219-14.2015

Copyright $\odot 2015$ the authors $\quad 0270-6474 / 15 / 351763-10 \$ 15.00 / 0$ courses of activity in different regions covary, the regions are considered functionally connected (Friston et al., 1993). Another approach involves measuring variations in connectivity associated with cognitive states or processes that fluctuate trial-by-trial (Friston et al., 1997; Rissman et al., 2004). This event-related approach is useful when studying memory using recognition memory tests, when successful retrieval occurs on an unpredictable subset of trials. For such tests, an approach that permits connectivity to be contrasted on a trial-wise basis enables the direct comparison of connectivity during successful and unsuccessful retrieval.

Assessing dynamic modulation of connectivity during episodic retrieval tests might provide insight into the neural mechanisms supporting recollection (Ranganath et al., 2005; Staresina et al., 2013). Although the brain regions engaged during successful recollection have been identified, recollection-sensitive interactions between members of this putative network, or with other brain regions, are not well characterized. Additionally, investigation of individual differences in recollection-related modulation of connectivity might provide insight into aspects of brain function that support accurate memory. In one previous study, taskrelated changes in connectivity between several large-scale brain networks were assessed during episodic retrieval relative to a control task (Fornito et al., 2012). Similarity between two of the networks in their task-related connectivity changes with the rest of the brain correlated across individuals with the response latencies, but not the accuracy, of recollection judgments. In another study (Schedlbauer et al., 2014), connectivity between a set of regions was reported to be denser for successful than unsuccessful contextual judgments. Additionally, connection density dur- 
ing successful retrieval was correlated across subjects with retrieval accuracy. However, it remains to be demonstrated whether changes in connectivity associated with successful relative to unsuccessful recollection covary with individual differences in memory accuracy.

Here, we applied psychophysiological interactions (PPI) analysis (Friston et al., 1997) to fMRI data acquired in three independent studies, each using a different operationalization of recollection. We investigated whether core recollection regions exhibited task-related changes in functional connectivity with other brain regions during successful compared with unsuccessful recollection, and whether the magnitude of these changes covaried across individuals with recollection accuracy.

\section{Materials and Methods}

Participants. Experiment 1 included 24 participants (13 female) age $19-29$ years $(\mathrm{M}=23.79$; $\mathrm{SD}=2.43)$. Experiment 2 included 36 participants (17 female) age $18-29$ years $(\mathrm{M}=22.2$; $\mathrm{SD}=3.0)$, and Experiment 3 included data from 28 participants ( 14 female), age $18-29$ years $(\mathrm{M}=23.14 ; \mathrm{SD}=3.34)$. Participants were recruited from the University of Texas at Dallas (UT Dallas) and surrounding communities. All were right-handed, had normal or corrected-to-normal vision, and had learned English before age five. They were compensated with $\$ 30 / \mathrm{h}$ for their participation. All participants gave informed consent according to the procedures approved by the UT Dallas and University of Texas Southwestern Institutional Review Boards.

Materials and procedures. Recollection was assessed using a different behavioral paradigm in each of the three experiments. Experiment 1 used a Remember/Know procedure (Tulving, 1985; Gardiner, 1988). Experimental items consisted of 216 pictures and 216 corresponding object names. Of the 216 picture-word pairs, 144 were randomly selected to be presented during the study phase. For 72 of the pairs, the picture was presented, whereas for the remainder the name was presented. At test, the 144 studied items and an additional 72 new items were presented as words only. During encoding, subjects made one of two judgments about the denoted object depending on whether it was presented as a picture or a word. The test task was to judge whether each test word corresponded to a studied item, regardless of its study format. There were three response options. Subjects were instructed to respond "Remember" on trials where recognition was accompanied by retrieval of a specific detail or details from the study episode. "Know" responses were to be used when an item was recognized in the absence of the retrieval of any specific detail about the study event. "New" judgments were to be given if a test item was not recognized from the study phase. For the present purposes, behavioral and fMRI data were analyzed after collapsing over the format of the studied items. Recollection success was operationalized by the contrast between the fMRI BOLD activity elicited during retrieval by studied items endorsed Remember and studied items endorsed Know.

Experiment 2 used an associative memory procedure. During the study phase, subjects studied 240 visually presented pairs of concrete words, judging which of the two denoted objects was more likely to fit into the other. At test, 160 of the studied word pairs were re-presented (intact pairs). A further 80 test pairs comprised studied words that had been repaired from study (rearranged pairs). There were also 80 new pairs, consisting of unstudied words. The retrieval task was to judge whether each test pair was "Intact" (studied together), "Rearranged" (both words were studied but on different trials), or New (neither word was studied). For the purpose of fMRI analyses, successful recollection was operationalized as the contrast between activity elicited by intact test pairs correctly judged as such, and that elicited by intact pairs wrongly judged as rearranged.

Experiment 3 involved a source memory task (data from this experiment, albeit subjected to quite different analyses from those described here, have been published previously by Elward et al., 2014). Experimental items consisted of 240 color pictures of objects. During the study phase, 160 of the objects were presented, 80 in association with the depiction of one type of coin (a Lira), and 80 with the depiction of another (a Deutschmark). The requirement was to make an "indoor/outdoor" judgment about each object. At test, each studied picture was represented along with the 80 unstudied pictures. The task was to judge whether each picture had been studied in association with a Lira or a Deutschmark or whether it was unstudied; correct source judgments were differentially rewarded according to the identity of the coin. Here, test items were collapsed across the two study contexts (sources) and recollection-related activity was operationalized by the contrast between fMRI responses to studied items attracting correct versus incorrect source judgments.

MRI data acquisition and preprocessing. In each experiment, MRI data were acquired on the same 3T Philips Achieva MRI scanner (Philips Medical Systems) equipped with a 32 channel receiver head coil. Functional images were acquired using a $\mathrm{T} 2^{*}$-weighted, BOLD echoplanar (EPI) sequence (SENSE factor 1.5, flip angle $70^{\circ}, 80 \times 80$ matrix, $\mathrm{FOV}=$ $24 \mathrm{~cm}, \mathrm{TR}=2000 \mathrm{~ms}, \mathrm{TE}=30 \mathrm{~ms})$ and T1-weighted anatomical images were acquired with a magnetization-prepared rapid gradient echo pulse sequence $\left(\mathrm{FOV}=240 \times 240,1 \mathrm{~mm}^{3}\right.$ isotropic voxels).

MRI data were preprocessed in SPM8 (Wellcome Department of Cognitive Neurology, London, UK). Functional scans were realigned to the mean EPI image, subjected to slice timing correction, reoriented to approximate the MNI reference template, spatially normalized to MNI space, and smoothed using an $8 \mathrm{~mm}$ full-width half-maximum Gaussian kernel. Anatomical images were similarly normalized to MNI space.

fMRI data analysis: average BOLD signal changes associated with successful recollection. Statistical Parametric Mapping (as implemented in SPM8) based on a General Linear Model (GLM) was used to analyze the fMRI data. At the individual subject level, a number of events of interest (the exact number depending on the experiment) were modeled; these always included one event corresponding to successful recollection and one event for unsuccessful recollection. The neural response elicited on each trial was modeled as a delta function that corresponded to the onset of each trial. Delta functions were then convolved with a canonical hemodynamic response function to model the predicted BOLD response. Other covariates entered into the first-level models included six parameters that represented the motion-related variance in the data (3 for rigid-body translation and 3 for rotation), as well as regressors representing each of the separate scan sessions. An autoregressive AR(1) model was used during parameter estimation to correct for time-series correlations in the data. Data were high-pass filtered with a cutoff of $128 \mathrm{~s}$ to remove any confounds due to slow signal drifts. Individual subjects' parameter estimates for successful and unsuccessful recollection events from these first-level analyses were entered into a group-level analysis that treated subjects as a random variable. Contrast images comparing activity associated with recollection to activity associated with familiarity were constructed. These images were thresholded at $p<0.001$, uncorrected, with a 23-voxel extent threshold (giving a cluster-wise corrected threshold of $p<0.05$ according to the Monte-Carlo simulation implemented in AFNI; http://afni.nimh.nih.gov/pub/dist/doc/manual/AlphaSim.pdf).

To compare results across experiments, first-level contrast images from each experiment were entered into a single second-level analysis, with experiment as a between-subjects factor. To identify regions that showed reliable recollection success effects across experiments, the main effect of recollection success was inclusively masked by the simple effect from each experiment $(p<0.01)$ to ensure that only voxels where a recollection effect was evident in all three experiments were included. To identify differences in recollection-related activity across experiments, we examined the main effect of experiment, thresholded at $p<0.001$ with a 23-voxel extent threshold.

fMRI data analysis: recollection-related changes in BOLD time-series correlations. PPI was used to identify recollection-related changes in connectivity (Friston et al., 1997). PPI identifies event-related differences in the time-series correlations (functional connectivity) between a "seed" region and the rest of the brain after partialling out the main effect of event-related changes in activity and event-independent connectivity. Five PPI analyses, one for each seed region of interest, were conducted for each experiment. Seed regions were selected based on the results of the univariate analyses described above and previous research implicating these regions in successful recollection (Spaniol et al., 2009; Kim, 2010; 
Rugg and Vilberg, 2013). Seed regions included left angular gyrus (AnG), medial prefrontal cortex (mPFC), hippocampus (Hipp), middle temporal gyrus (MTG), and posterior cingulate cortex (PCC). To create the PPI interaction term of interest, we created a task vector that coded successful recollection trials as 1 , unsuccessful recollection trials as -1 , and all other trials as 0 . We then extracted a time course from each seed region in a subject-specific manner. We did this by creating functionally defined masks for each seed region, based on the results of the group-level univariate recollection success contrast. Masks comprised 10-mm-radius spheres centered on the peak of the group-level recollection effect within each seed region. The hippocampal mask was additionally constrained by an anatomical mask of the hippocampus, which was defined according to the AAL atlas (Tzourio-Mazoyer et al., 2002). The anatomical constraint on the hippocampal mask ensured that voxels that were within a $10 \mathrm{~mm}$ radius sphere of the peak but were outside of the hippocampus were excluded from analysis, which was not a concern for any of the other seed regions. After these masks were created, we determined the peak recollection effect within these masks for each subject. We drew 3-mm-radius spherical regions-of-interest (ROIs) centered on these peaks, and extracted the representative time course (first eigenvariate) of the included voxels. The time courses were then deconvolved with the canonical hemodynamic response function (HRF) in SPM8, multiplied by the event structure vector, and then reconvolved to provide the PPI term that was entered into the first-level regression model as the regressor of interest. The event structure vector was convolved with the HRF and entered into the regression model as the psychological (task) regressor of no interest, along with the original time course from the seed region of interest. Additional nuisance covariates included the same motion and session parameters that were also entered into the mean signal analyses, and data were again subjected to a high-pass filter with a cutoff of $128 \mathrm{~s}$.

Parameter estimates of the PPI regressor from the first-level PPI GLM analyses were brought to the second level where separate group-level, random effects analyses were performed for each seed in each experiment. One-sample $t$ tests identified voxels where connectivity with a given seed was higher during successful versus unsuccessful recollection. The resulting contrast images from each of these analyses were thresholded at $p<0.001$, uncorrected, with a voxel extent threshold of 23 (see previous section). To identify brain regions where recollection-related increases in connectivity were reliable across experiments, first-level contrast images from each experiment were entered into a single, secondlevel model, with experiment as a between-subjects factor. The main effect across experiments was thresholded at $p<0.001$ (23 contiguous voxels) and inclusively masked by the simple effect of each experiment (inclusive mask threshold of $p<0.01$; see above).

Behavioral correlations with recollection-related changes in connectivity. To determine the extent to which recollection-related increases in connectivity were related to recollection accuracy, we calculated the correlation between recollection accuracy $(p \mathrm{R})$ and connectivity change between each of the five seed regions and 90 target regions that were defined based on the AAL atlas. We used the AAL atlas (TzourioMazoyer et al., 2002) to parcellate each subject's brain into 90 cortical, striatal, and thalamic regions in a manner unbiased by the outcomes of the fMRI analyses. Using the MarsBar toolbox in SPM8 (Brett et al., 2002), we created mask images corresponding to each region. Then, for each seed region, we extracted the mean parameter estimate of connectivity change across all voxels within a given ROI, for each subject. We constructed correlation matrices where each column represented a different seed and each row represented one of the 90 "target" regions defined according to the AAL atlas. Using the parameter estimates that were extracted for each subject, we calculated for each seed-target pair a between-subjects Pearson's correlation coefficient that reflected the strength and direction of the relationship between recollection-related changes in connectivity and recollection accuracy. In each experiment, for every seed we calculated both the average size of the correlations across all target regions, and the proportion of statistically significant correlations $(p<0.05$, two-tailed).

Permutation analyses tested whether, collapsed across all target regions, the average size and number of these correlations were greater than would be expected by chance. For each experiment, we randomly scram- bled both the behavioral measures and the parameter estimates for each seed-target pair and then constructed correlation matrices in the same way that was done with the actual data. We repeated this process 1000 times per seed region for each experiment, constructing two separate null distributions for each seed region/experiment. The first null distribution reflected the average of the correlations between a given seed region and all target regions that should be expected by chance. From this null distribution, we calculated the chance probability of obtaining the observed average correlation value for each seed region by computing the proportion of iterations within the null distribution that exceeded the obtained value. The second null distribution was with respect to the proportion of significant correlations for each seed region that would be expected by chance. We then calculated the number of iterations within the null distribution for which the total number of significant $r$ values exceeded the observed number of significant values.

\section{Results}

\section{Behavioral results}

Different behavioral indices were used to estimate the probability of recollection $(p \mathrm{R})$ in each experiment. In Experiment $1, p \mathrm{R}$ was defined as the difference between the proportions of studied and new items given a Remember response (Jacoby, 1991). The mean score was $0.59(\mathrm{SD}=0.11)$, significantly above the chance value of zero $\left(t_{(23)}=25.76, p<0.001\right)$. In Experiment $2, p \mathrm{R}$ was measured as the difference between the proportion of intact pairs correctly judged intact and the proportion of rearranged pairs wrongly judged as intact. Mean $p \mathrm{R}$ was $0.48(S D=0.19)$, again significantly greater than chance $\left(t_{(35)}=15.09, p<0.001\right) . p \mathrm{R}$ in Experiment 3 was estimated as the proportion of studied items attributed to the correct study context relative to all studied items recognized as old. Mean source accuracy (i.e., $p \mathrm{R}$ ) across subjects was $0.65(\mathrm{SD}=0.11)$, also significantly greater than chance $\left(t_{(27)}=\right.$ $7.01, p<0.001)$.

\section{Average BOLD signal changes associated with successful recollection}

To identify regions demonstrating reliably greater neural activity (as indexed by fMRI BOLD signal) for trials associated with successful relative to unsuccessful recollection we examined the main effect of successful recollection, collapsing across experiments (thresholded at $p<0.001$, uncorrected, voxel extent $=$ $23)$, masked by the simple effect of successful recollection from each experiment $(p<0.01)$. We first identified regions demonstrating greater neural activity for trials associated with successful relative to unsuccessful recollection in each experiment (see Materials and Methods). Whole-brain results of the masked main effect are presented in Figure 1. The masked main effect included a set of regions that correspond closely with those held to constitute the core recollection network (see Introduction). The regions included left AnG, bilateral Hipp/parahippocamal cortex, $\mathrm{mPFC}$, and PCC. In addition, recollection effects were evident in left MTG, along with right ventrolateral prefrontal cortex (VLPFC), right supplementary motor area (SMA), the striatum, and the cerebellum.

We used the main effect of experiment from the foregoing ANOVA model to identify regions where recollection effects differed according to experiment (see Materials and Methods). Differences were limited to a few small clusters, including ones in right VLPFC, bilateral dorsolateral prefrontal cortex (DLPFC), left middle occipital gyrus, and right angular gyrus. In each case, the effect was driven by greater recollection-related activity in Experiments 1 and 3 compared with Experiment 2. Across all experiments and seeds, no regions were identified where connectivity was greater during unsuccessful relative to successful recollection. 

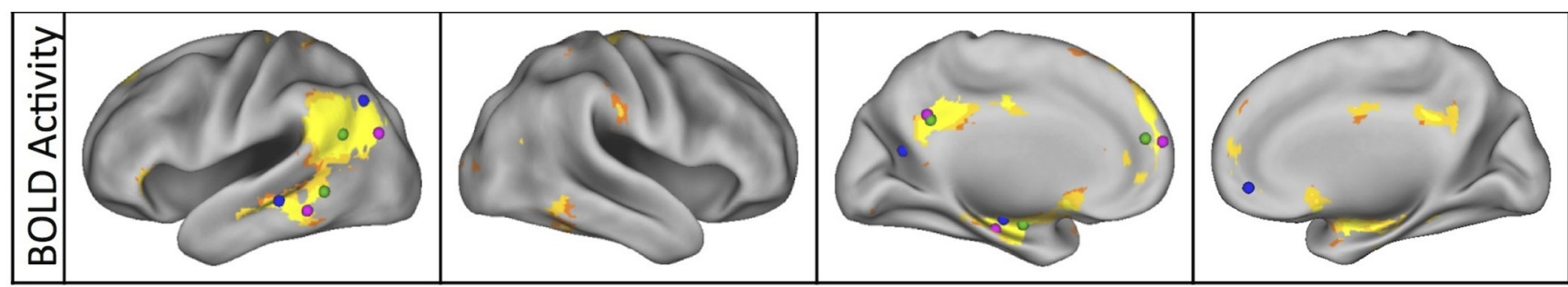

Exp. $1 \bigcirc$ Exp. $2 \bigcirc$ Exp. 3

Figure 1. Regions where the magnitude of the BOLD signal was significantly greater during successful relative to unsuccessful recollection across all three experiments. The main effect of successful retrieval activity across experiments was thresholded at $p<0.001$ with a voxel extent of 23 and then masked by the simple effect from each experiment at $p<0.01$. Effects are projected onto left and right hemisphere inflated surface caret brains (Caret5; Van Essen et al., 2001) and presented from medial and lateral views. The group peaks of activity within each seed region (AnG, mPFC, Hipp, MTG, PCC; see Materials and Methods) that were used as masks to identify individual subject peaks that were then used to define seed regions are depicted by foci projected onto the lateral and medial surfaces of the caret brains (Experiment 1, magenta; Experiment 2, green; Experiment 3, blue).

\section{Recollection-related changes in inter-regional connectivity}

To identify brain regions that demonstrated recollection-related increases in functional connectivity, we ran five separate PPI analyses, one for each of five seed ROIs, for each experiment (for a total of 15 separate analyses). Seed regions comprised different components of the core recollection network, namely, the left AnG, mPFC, PCC, and Hipp. In light of the consistency with which it has been reported to demonstrate recollection effects (Spaniol et al., 2009; Hayama et al., 2012; Vilberg and Rugg, 2012, 2014), we also included the left MTG as a seed region (see Materials and Methods). The masked main effects identifying regions where there were significant and consistent recollection-related increases in connectivity across experiments (main effect thresholded at $p<0.001$, uncorrected, voxel extent $=23$ masked by the simple effect from each experiment thresholded at $p<0.01)$ are depicted for each seed region in Figure 2. As is evident from the figure, increased connectivity for successful relative to unsuccessful recollection was apparent for all seed regions. Connectivity was found to increase both with other members of the core recollection network, such as the AnG, MTG, and PCC, and regions that are not typically considered part of the network, such as the left DLPFC, dorsal aspects of the lateral PCC, dorsal ACC (dACC), and extrastriate visual cortex.

We tested for any differences between seed regions or experiments in recollection-related connectivity changes by entering the first-level contrast images into a single second-level, two-way, mixed-factor ANOVA model (between-subjects factor of experiment, and within-subjects factor of seed region). The ANOVA failed to identify an experiment by seed region interaction anywhere in the brain. A few small clusters did however demonstrate a main effect of experiment. These were mainly localized to visumedial and lateral views.

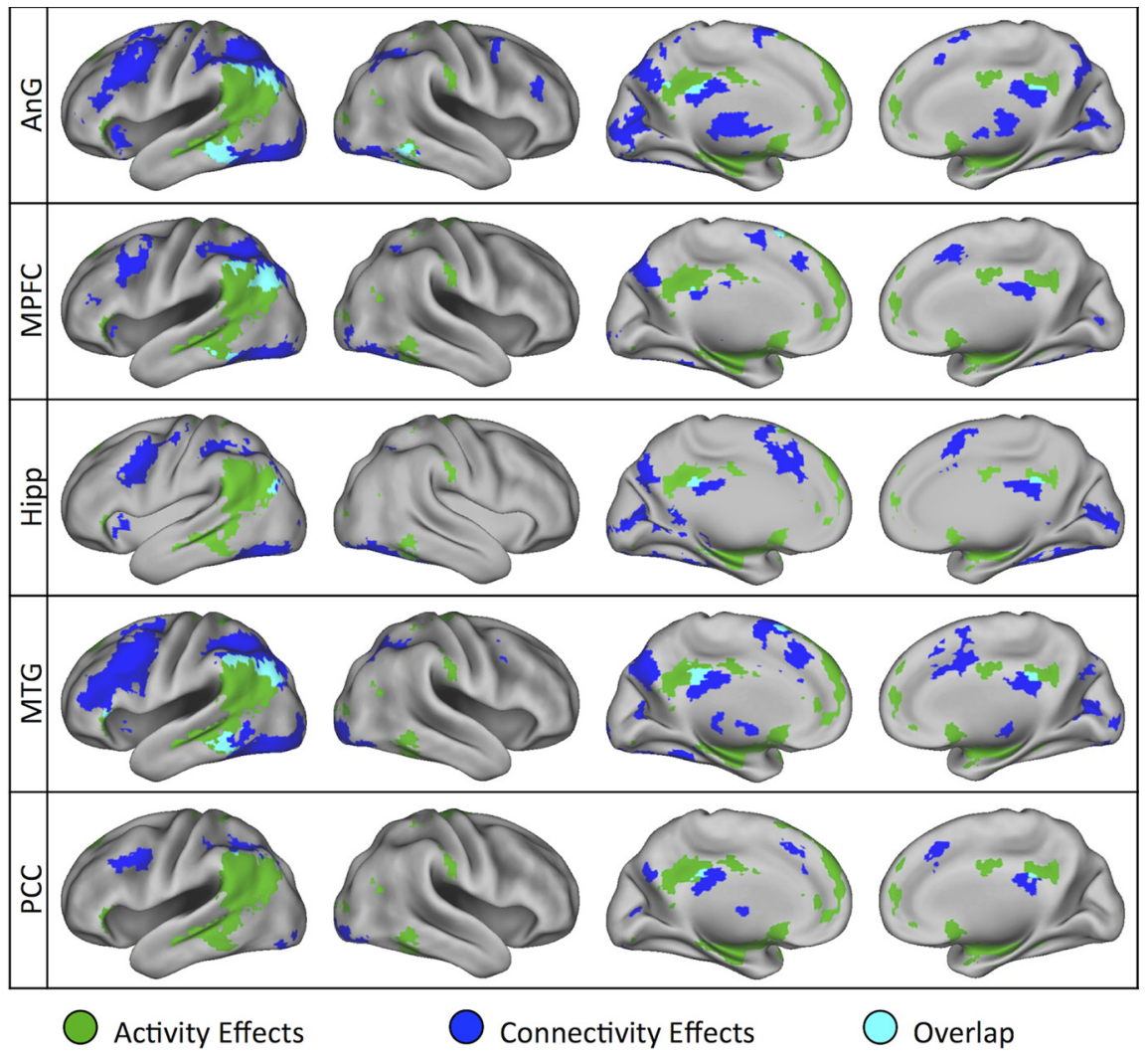

Figure 2. Overlap map of recollection-related activity and connectivity effects. Regions where there was a reliable recollectionrelated change in activity are depicted in green. Regions where there was a reliable recollection-related change in connectivity with a given seed region are depicted in blue. Regions that demonstrated both a recollection-related change in activity and a recollection-related change in connectivity with a given seed region are depicted in cyan. Main effects across experiments were thresholded at $p<0.001$ with a voxel extent of 23 and then masked by the simple effect from each experiment at $p<0.01$. Effects are projected onto left and right hemisphere inflated surface caret brains (Caret5; Van Essen et al., 2001) and presented from ally responsive occipital and fusiform cortex, along with a cluster in the left SMA. In each case, the effect was driven by greater connectivity changes in Experiment 1 compared with Experiment 3. The ANOVA also identified a main effect of seed region, but again, only in a few small clusters throughout the brain. Regions demonstrating this effect included bilateral occipitoparietal cortex, left inferior temporal cortex, left precentral gyrus, right inferior frontal gyrus, PCC, and bilateral cerebellum. Although 

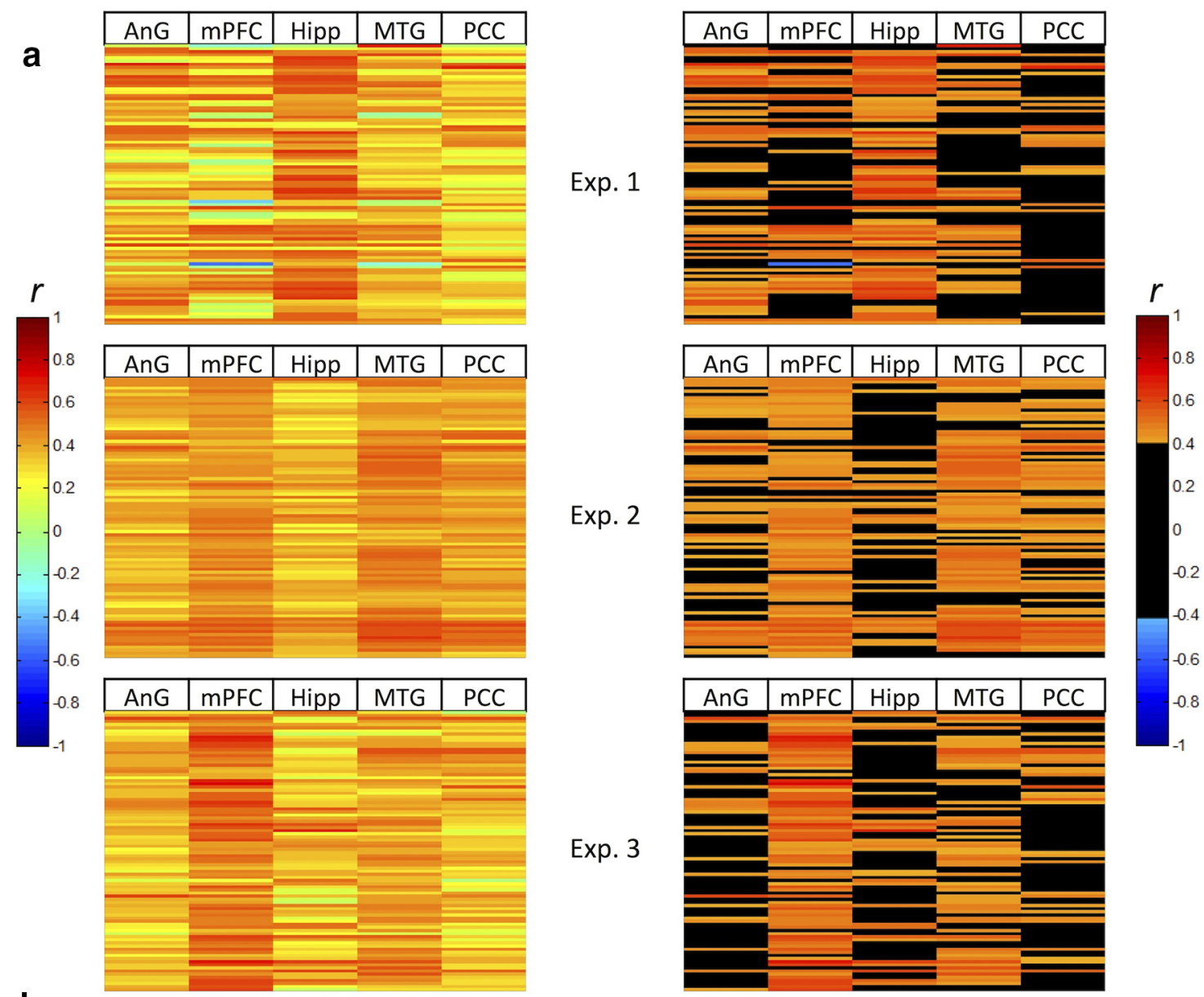

b
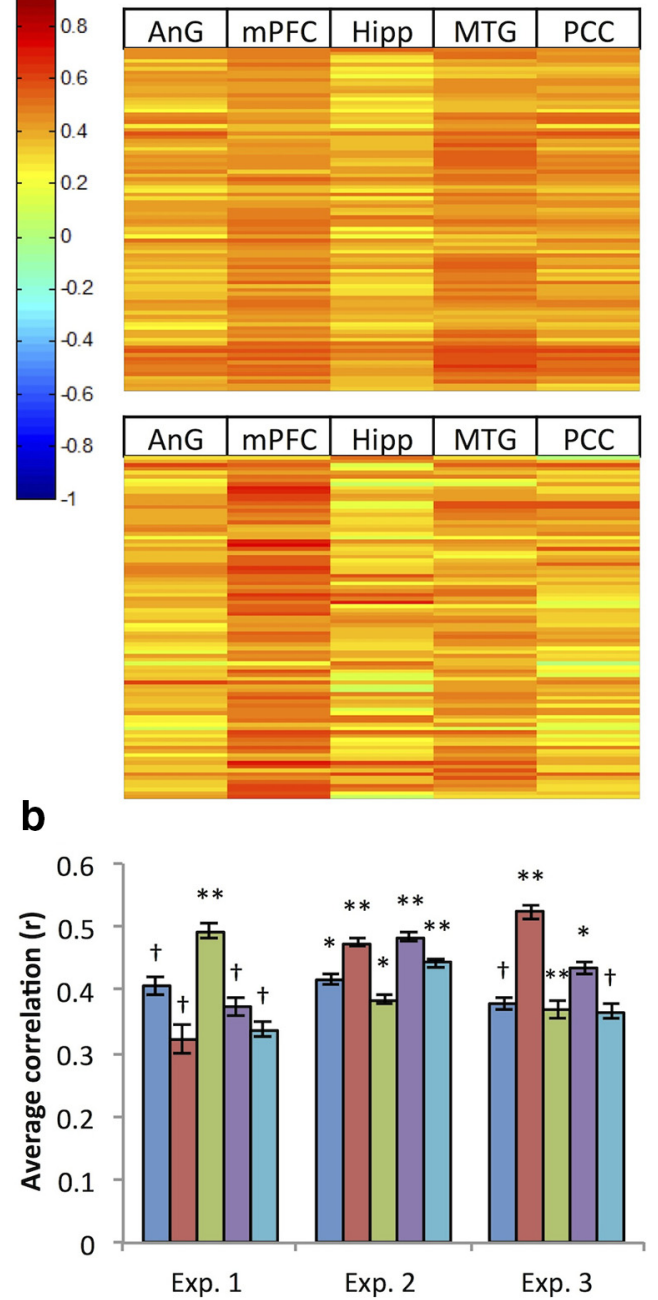

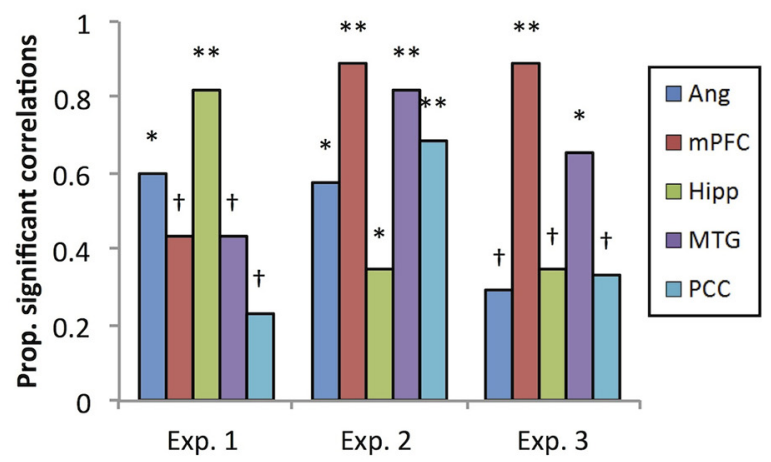

Figure 3. Correlations between recollection-related increases in connectivity and recollection accuracy. $\boldsymbol{a}$, The depicted matrices represent the size of the correlation between seed-target connectivity and behavioral performance across five seeds and three experiments. Within each matrix, each column corresponds to one of the five seed regions for which a PPI analysis was conducted. Each row of the matrix corresponds to one of the 90 target regions defined by the AAL atlas. Colors within each cell of the matrix represent the size of the correlation (Pearson's $r$ ) between recollection accuracy and the recollection-related increase in connectivity associated with each seed-target pair. Matrices correspond to Experiments 1 (top), 2 (middle), and 3 (bottom). Matrices on the left and right are identical except that those on the left are coded continuously, whereas those on the right are thresholded at $r>0.41$ ( $p<0.05$ for the experiment with the smallest sample size). $\boldsymbol{b}$, Bar graphs represent the average correlation between recollection-related increases in connectivity and recollection accuracy for each seed region (left), and the proportion of brain regions where the change in connectivity correlated with recollection accuracy with an effect size of $r>0.41$ (right). Error bars reflect the SEM; $† p<0.05,{ }^{*} p<0.01,{ }^{* *} p<0.001$.

the pattern of results driving this effect differed for each cluster, in general, estimates of changes in connectivity tended to be greater for the AnG and MTG seed regions relative to the other seed regions.

\section{Relationship between recollection-related changes in} connectivity and recollection accuracy

We next investigated whether the magnitude of recollectionrelated increases in connectivity in the different seed regions co- varied across subjects with recollection accuracy. For each experiment, we constructed seed-target correlation matrices, where each column of the matrix represented one of the five seed regions, and each row represented one of the 90 target regions defined by the AAL atlas (see Materials and Methods). For each seed-target pair, we extracted the mean parameter estimate of connectivity change for every subject. We then calculated the across-subjects correlation between recollection accuracy and recollection-related connectivity change for each seed-target 
pair. The resulting correlation matrices are depicted in Figure $3 a$. To quantify the strength of the relationship between connectivity changes and recollection accuracy, for each seed, we calculated the average correlation coefficient across all target regions (Fig. $3 b)$. Permutation analyses tested whether these values were significantly greater than what would be expected by chance (see Materials and Methods). The strongest results were in Experiment 2, where the average size of the correlation exceeded chance $(p<0.01$, which corresponds to a Bonferroni-corrected $\alpha$-level of $p<0.05$, after correcting for 5 comparisons) in all five seed regions. In Experiment 3, the average correlation was significant for the $\mathrm{MPFC}$, Hipp, and MTG seeds, and it was also significant in the AnG and PCC seeds before Bonferroni correction $(p<0.05$, uncorrected). In Experiment 1, the average correlation across target regions exceeded chance only for the Hipp seed region after Bonferroni correction; however, without this correction, it exceeded chance $(p<0.05)$ for all five seed regions.

We also calculated the proportion of brain regions where the change in connectivity correlated with recollection accuracy with an effect size of $r=>0.41$ (Fig. $3 b$ ). This effect size was chosen because it represents the smallest statistically significant value $(p<0.05$, two-tailed) in the experiment with the smallest sample size (Experiment 1). Permutation analyses were again used to determine whether these proportions exceeded chance (see Materials and Methods). In Experiment 2, the proportions of correlations exceeding our chosen threshold were greater than chance in all five seed regions $(p<0.01)$. In Experiment 3, proportions were significant for the $\mathrm{mPFC}$ and MTG seed regions after correcting for multiple comparisons $(p<0.01)$, and for each of the remaining seeds (AnG, Hipp, and PCC) without this correction $(p<0.05)$. In Experiment 1, proportions were significant for the AnG and Hipp seeds only after correcting for multiple comparisons $(p<0.01)$, but without this correction, the proportions significantly exceeded chance for all of the seed regions $(p<$ $0.05)$. Together, these analyses indicate that recollection-related changes in connectivity between each of the five seed regions and much of the cortex were reliably correlated with behavioral performance. We created heat maps for each seed region to visualize the target regions where the correlations between connectivity change and performance were most consistent (Fig. 4). As is evident from the figure, the correlation between accuracy and connectivity exceeded $r=>0.41$ in at least two experiments in the majority of seed-target pairs, with this relationship occurring in all three experiments in several seed-target pairs, especially for the AnG, mPFC, and PPC seeds. Although the regions demonstrating correlations between connectivity and performance were diffusely spread throughout the brain, regions where this relationship was observed most consistently across experiments were mPFC, PCC, left AnG, superior frontal gyrus, and middle occipital gyrus.

\section{Comparison and control analyses}

We ran several additional analyses to rule out possible confounds with respect to the correlations between connectivity change and memory performance described above, and to narrow down the range of potential explanations.

\section{Correlations between $\mathrm{p} R$ and recollection-related changes in BOLD activity}

We examined the relationship between recollection accuracy and the recollection-related increase in BOLD activity in both target and seed regions. Unlike the PPI effects, recollection-related increases in BOLD signal were not reliably correlated with memory performance. Unsurprisingly, in light of these findings, when the correlations between connectivity change and memory performance were re-estimated after controlling for BOLD signal change in the both seed and target regions, the results were almost identical to those from the original analyses.

\section{Correlations between recollection-related changes in connectivity and other behavioral measures}

To assess the specificity of the relationship between connectivity and recollection accuracy, we investigated whether recollectionrelated changes in connectivity correlated with other performance measures, including reaction time (RT; cf. Fornito et al., 2012), and estimates of familiarity and response bias. RT was defined as the average response latency to all test items. In Experiment 1, familiarity was estimated according the procedure recommended by Yonelinas and Jacoby (1995) [pKhit/(1-pRhit) pKfalse alarm/(1 - pRfalse alarm $)]$. In Experiment 2 , familiarity was defined as $p$ Rearranged $\mid$ Intact $/(p$ Rearranged $\mid$ Intact $+p$ New $\mid$ Intact $)-p$ Rearranged $\mid$ New $/(p$ Rearranged $\mid$ New $+p$ New $\mid$ New $)$. In Experiment 3, familiarity was defined as $\mathrm{pSI} /(1-p \mathrm{SC})$, where $p \mathrm{SI}$ is the proportion of incorrect source judgments and $p S C$ is the proportion of source correct judgments. Bias was assessed in Experiments 1 and 2 only, as in Experiment 3, all behavioral and neural measures were collapsed across the two possible source judgments. In Experiment 1, bias was calculated as $p$ Rfalse alarm/( $p$ Rfalse alarm + pKflase alarm). In Experiment 2, bias was defined as $p$ Intact Rearranged/( $p$ Intact $\mid$ Rearranged $+p$ Rearranged $\mid$ Rearranged $)$.

To test whether there was a relationship between recollectionrelated changes in connectivity and the foregoing measures of behavioral performance, we ran a series of analyses identical to those used for recollection accuracy, but with the recollection accuracy data replaced by RT, familiarity, and bias respectively. Permutation analyses revealed that for each of these measures, neither the average size of the correlations nor the proportion of significant correlations exceeded chance. We also re-estimated the recollection accuracy correlation matrices after partialling out the effects of RT, familiarity, and bias. In each case, the results closely resembled those from the original analyses. Hence, these findings demonstrate that the relationship between accuracy and recollection-related connectivity change cannot be explained by variance in RT, familiarity, or bias; rather, the relationship between changes in connectivity and performance are specific to recollection accuracy.

\section{Correlations between recollection-related changes in connectivity} and $\mathrm{p} R$ controlling for differences in the proportion of trials associated with successful and unsuccessful recollection

In each experiment, individuals with better recollection performance tended to have a higher ratio of successful to unsuccessful recollection trials than did worse performers. Thus, across subject differences in the relative proportions of trials in the two response categories might be responsible for mediating the relationship between connectivity change and performance. To assess this possibility, we reanalyzed the data after randomly selecting subsets of trials to equate the number of successful and unsuccessful recollection trials for each subject. The relationships between connectivity change and recollection accuracy were largely unaffected. Hence, the relationship is not mediated by across-subject variation in relative trial numbers.

Correlations between $\mathrm{p} R$ and item-related connectivity change In a final analysis, we investigated whether the relationship between recollection-related connectivity increases and recollection accuracy was a manifestation of a more general relationship 


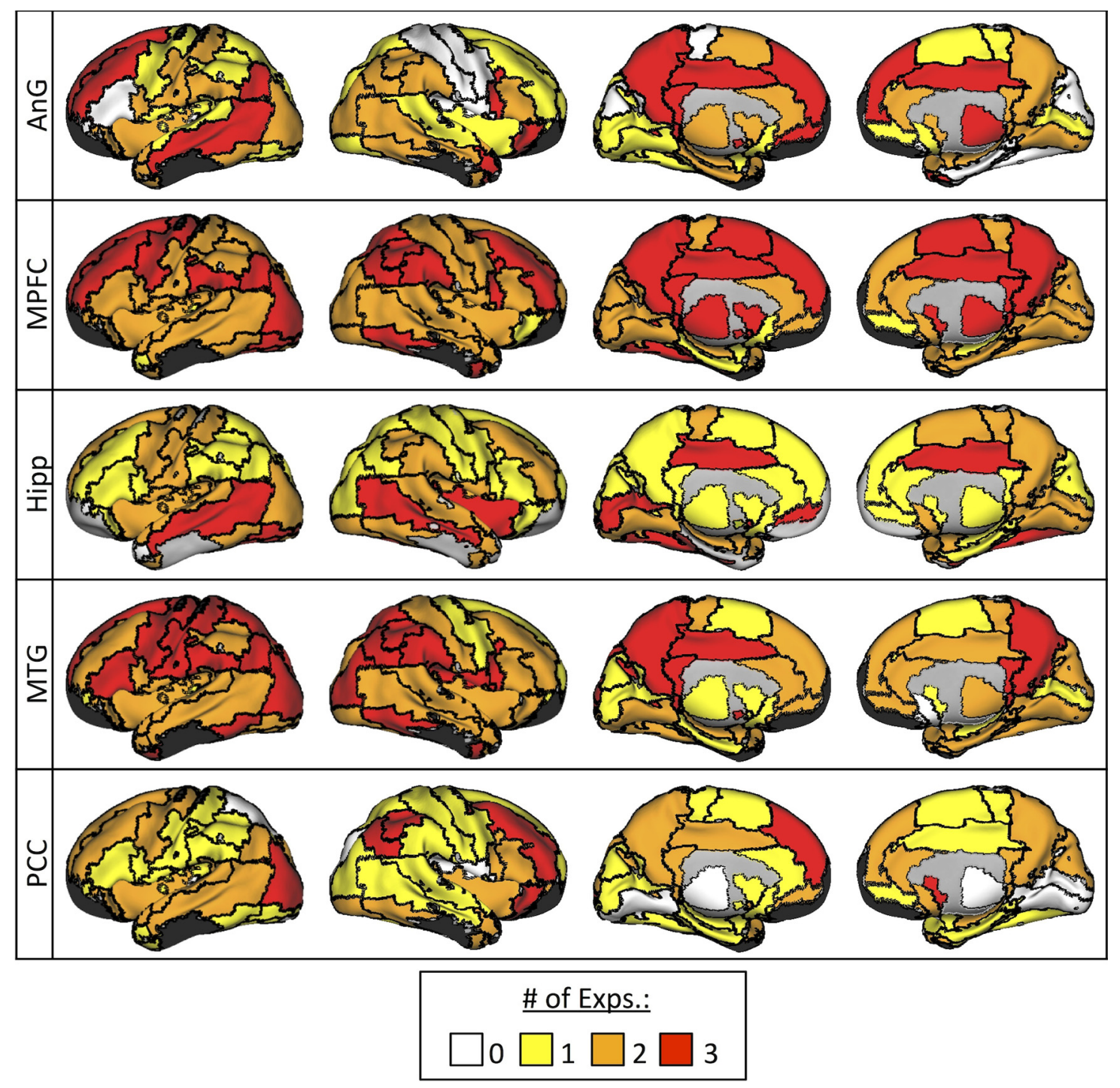

Figure 4. Heat maps representing, for each of the $90 \mathrm{ROls}$, the number of experiments for which there was a significant relationship between connectivity change and recollection accuracy. Borders of the 90 cortical, striatal, and thalamic regions defined by the AAL atlas are projected onto left and right hemisphere inflated surface caret brains (Caret5; Van Essen et al., 2001) which are presented from medial and lateral views. For each seed, target regions where the correlation between connectivity and accuracy was significant in all three experiments are presented in red; regions where this relationship was significant in two of three experiments are presented in orange; regions where this relationship was significant in only one of three experiments are presented in yellow, and regions where this relationship was not significant in any of the three experiments are presented in white. Correlations were considered statistically significant if the correlation coefficient (Pearson's $r$ ) exceeded $r>0.41$. This effect size was chosen because it corresponds to the smallest statistically significant value $(p<0.05$, two-tailed) in the experiment with the smallest sample size.

between brain dynamics and performance. By this hypothesis, the brains of higher performing individuals are simply more capable, in general, of switching between different states or network configurations than those of lower performing individuals. We tested this hypothesis by running additional PPI analyses in which the task regressor (and consequently the PPI regressor) was constructed by weighting every test trial as 1 , thereby generating a PPI that represented where connectivity increased relative to baseline each time a test item was presented. The same analysis procedures that identified the relationship between recollectionrelated connectivity increases and performance failed to identify any such relationship for this more general measure of itemrelated connectivity change.

\section{Discussion}

In three experiments using different operational definitions of recollection, brain regions exhibiting recollection-related increases in BOLD activity also demonstrated increased functional connectivity both with other members of the core recollection network and with regions extrinsic to the network, notably the DLPFC and intraparietal sulcus, where BOLD activity did not vary with recollection success. These findings add to previous work pointing to the significance of increased connectivity in the context of episodic memory retrieval (Staresina et al., 2013; Watrous et al., 2013; Schedlbauer et al., 2014). In addition, the magnitude of recollection-related increases in connectivity covaried across subjects with recollection accuracy. Although prior studies have reported relationships between memory performance and low-frequency fluctuations in the BOLD signal during sustained periods of task engagement or rest (Wig et al., 2008; Hampson et al., 2010; Tambini et al., 2010; Wang et al., 2010; but see, Schedlbauer et al., 2014), the present findings constitute novel evidence that recollection-related modulation of connectivity can also serve as a predictor of individual differences in recollection accuracy. 
The results from the three datasets were highly consistent, with respect to both the regions where recollection-related increases in activity and connectivity were identified, and where increases in connectivity correlated with performance. Whereas some differences in the pattern of connectivity effects across experiments were identified, we focus below on features of the data that converged across experiments. This is not to suggest that the across-experiment differences that we observed are unimportant. It is possible, for example, that the findings that connectivity increases in fusiform cortex were greater in Experiment 1 than Experiment 3 reflects between-experiment differences in the relevance of retrieved visual information for the respective memory tests. Similarly, the finding that the hippocampal seed demonstrated the strongest correlation with recollection performance in Experiment 1, whereas the strongest correlations were found for the mPFC and MTG seeds in the other experiments, might signify that the three regions were engaged differentially across experiments. At present, however, there is no means to determine which of the numerous variables that differed between the experiments were responsible for these or other differences in the pattern of the results.

Consistent with prior studies (for review, see Spaniol et al., 2009; Kim, 2010; Rugg and Vilberg, 2013) several regions that showed enhanced recollection-related activity in the current experiments corresponded with those held to constitute a core recollection network, including left AnG, mPFC, PCC, and Hipp, as well as the left MTG. When each of these regions was used as a seed, recollection-related connectivity increases were consistently identified both with other members of this network (although see below), and regions outside of the network, including DLPFC, dorsal aspects of the lateral parietal cortex, dACC, and extrastriate visual cortex. These findings are consistent with the results of two previous studies (Watrous et al., 2013; Schedlbauer et al., 2014), both of which identified more connections between a broadly distributed set of regions during successful than unsuccessful recall of contextual information. Here, we demonstrate not only that the number of inter-regional connections, but also the strength of those connections, varies with recollection success. Together, the present and prior findings point to the importance of both global and regionally specific connectivity changes during episodic memory retrieval.

Among the regions extrinsic to the core recollection network that demonstrated enhanced connectivity with members of the network were components of what has been termed the "executive metasystem" (Cocchi et al., 2013), which encompasses the "frontoparietal" and "cingulo-opercular" networks (Seeley et al., 2007; Dosenbach et al., 2008; Power et al., 2011; Power and Petersen, 2013). Regions belonging to these networks are held to act as flexible hubs that couple with different functional networks depending on task demands (Chadick and Gazzaley, 2011; Cole et al., 2013). Hence, recollection-related modulation of functional connectivity between these control networks and the core recollection network might reflect the allocation of control processes to the support of postretrieval operations, such as, evaluation of the products of recollection and the selection of the appropriate response (Rugg, 2004; Achim and Lepage, 2005; Ranganath et al., 2007). Thus, brain regions where recollectionrelated changes in signal magnitude are not detected might nonetheless contribute to retrieval processing by interacting with regions that do demonstrate a mean signal change.

In addition to regions belonging to control networks, recollection-related connectivity increases were also consistently identified in extrastriate visual cortex. Given the visual nature of the memoranda used in the three experiments (i.e., pictures and names of concrete objects), it is perhaps unsurprising that extrastriate visual areas, where "reinstatement" of retrieved visual information would be expected (for review, see Danker and Anderson, 2010), interacted with core recollection regions during successful recollection.

Regardless of the region used as a seed, neither the mPFC nor the hippocampus was identified as showing enhanced recollection-related connectivity. However, when these two regions were themselves used as seeds, they identified similar patterns of recollection-related connectivity increases to those identified for the other seed regions. Such "unidirectional" or "asymmetric" relationships are not uncommon in PPI analyses (Gerchen et al., 2014). Although it is tempting to interpret such asymmetries as evidence for effective connectivity (that is, a causal influence) between seed and target regions, there are several alternative explanations that can account for this pattern of results (e.g., differential colinearity between task and PPI regressors in different seed regions). Therefore, it would be premature to draw inferences about the directionality of the flow of information based on such asymmetries.

The current findings not only demonstrated that a number of brain regions showed enhanced recollection-related connectivity with members of the core recollection network, but also that the magnitude of connectivity change throughout much of the cortex correlated across individuals with recollection accuracy. Although recollection-related increases in connectivity, as identified at the group level, were relatively restricted anatomically, regions where the magnitude of connectivity increases correlated with performance were more broadly distributed. Particularly compelling evidence for the anatomically diffuse relationship between connectivity changes and performance comes from the finding that, in each of the 90 anatomically defined ROIs, a reliable correlation between connectivity and performance was evident for at least three of the seed regions across the different experiments (Fig. 4). Thus, the relationship between recollection-related modulation of connectivity and recollection accuracy might depend on a mechanism distinct from that responsible for the group-level, recollection-related connectivity enhancements evident in Figure 2.

It seems reasonable to suppose that the present recollection-related enhancements in connectivity reflect increases in inter-regional information exchange. However, what might be responsible for the anatomically diffuse nature of the relationship between connectivity change and memory performance? One possible explanation is related to the fact that an increase in functional connectivity between two brain regions can reflect not only increased inter-regional communication, but also the influence on multiple regions of a common, "driving" input (or inputs; Friston, 2011; Smith et al., 2011). From this perspective, the changes in connectivity that covary with behavioral performance might reflect a generic mechanism that modulates inter-regional synchrony across much of the brain. One possibility is that these effects are caused by event-related variations in the influence of one or more of the ascending neuromodulatory systems that innervate much of the cortex and other forebrain structures (Schölvinck et al., 2010; Lee and Dan, 2012). According to this hypothesis, the strength of any such neuromodulatory influence covaries with the strength of the recollection "signal," and hence with across-trial estimates of recollection performance. Importantly, the present data do not allow for an assessment of the causal relationship between these two variables; thus, it is unclear whether the strength of the putative modulatory input contributes to recollection accuracy, or whether strength of recollection influences cortical synchrony. 
In summary, the present findings suggest that coordinated functional interactions between distributed brain regions form part of the neural mechanism supporting successful recollection. Regions where activity varied as a function of recollection success demonstrated enhanced recollection-related connectivity with a consistent set of brain regions, including both regions belonging to and regions extrinsic to the core recollection network. This finding suggests that in addition to the core recollection network, other regions might contribute to recollection despite their failure to demonstrate changes in signal magnitude. The findings also demonstrated that the magnitude of connectivity change in widely distributed brain areas correlated across individuals with recollection accuracy, leading to the conjecture that these changes reflect a transient neuromodulatory input, which influences synchronicity throughout much of the brain. The finding that connectivity, unlike BOLD signal amplitude, covaried across subjects with recollection accuracy suggests that it may have promise for the understanding of individual differences in memory accuracy. Future research will be necessary to determine whether these dynamic changes in connectivity, and their relation with behavioral performance, are specific to the domain of episodic memory. Regardless, the current results highlight the value of examining event-related modulations of functional connectivity, in addition to modulation of local signal amplitude, to gain a more complete understanding of the neural correlates of successful memory retrieval.

\section{References}

Achim AM, Lepage M (2005) Dorsolateral prefrontal cortex involvement in memory post-retrieval monitoring revealed in both item and associative recognition tests. Neuroimage 24:1113-1121. CrossRef Medline

Brett M, Anton JL, Valabregue R, Poline JB (2002) Region of interest analysis using an SPM toolbox. Presentation at the 8th International Conference on Functional Mapping of the Human Brain, June 2-6, Sendai, Japan.

Chadick JZ, Gazzaley A (2011) Differential coupling of visual cortex with default or frontal-parietal network based on goals. Nat Neurosci 14:830 832. CrossRef Medline

Cocchi L, Zalesky A, Fornito A, Mattingley JB (2013) Dynamic cooperation and competition between brain systems during cognitive control. Trends Cogn Sci 17:493-501. CrossRef Medline

Cole MW, Reynolds JR, Power JD, Repovs G, Anticevic A, Braver TS (2013) Multi-task connectivity reveals flexible hubs for adaptive task control. Nat Neurosci 16:1348-1355. CrossRef Medline

Danker JF, Anerson JR (2010) The ghosts of brain states past: remembering reactivates the brain regions engaged during encoding. Psychol Bull 136: 87-102. CrossRef Medline

Dosenbach NU, Fair DA, Cohen AL, Schlaggar BL, Petersen SE (2008) A dual-networks architecture of top-down control. Trends Cogn Sci 12:99105. CrossRef Medline

Elward RL, Vilberg KL, Rugg MD (2014) Motivated memories: effects of reward and recollection in the core recollection network and beyond. Cereb Cortex. Advance online publication. Retrieved May 28, 2014. CrossRef Medline

Fornito A, Harrison BJ, Zalesky A, Simons JS (2012) Competitive and cooperative dynamics of large-scale brain functional networks supporting recollection. Proc Natl Acad Sci U S A 109:12788-12793. CrossRef Medline

Fox MD, Snyder AZ, Vincent JL, Corbetta M, Van Essen DC, Raichle ME (2005) The human brain is intrinsically organized into dynamic, anticorrelated functional networks. Proc Natl Acad Sci U S A 102:9673-9678. CrossRef Medline

Friston KJ (2011) Functional and effective connectivity: a review. Brain Connect 1:13-36. CrossRef Medline

Friston KJ, Frith CD, Liddle PF, Frackowiak RS (1993) Functional connectivity: the principal-component analysis of large (PET) data sets. J Cereb Blood Flow Metab 13:5-14. CrossRef Medline

Friston KJ, Buechel C, Fink GR, Morris J, Rolls E, Dolan RJ (1997) Psycho- physiological and modulatory interactions in neuroimaging. Neuroimage 6:218-229. CrossRef Medline

Gardiner JM (1988) Functional aspects of recollective experience. Mem Cognit 16:309-313. CrossRef Medline

Gerchen MF, Bernal-Casas D, Kirsch P (2014) Analyzing task-dependent brain network changes by whole-brain psychophysiological interactions: a comparison to conventional analysis. Hum Brain Mapp 35:5071-5082. CrossRef Medline

Greicius MD, Krasnow B, Reiss AL, Menon V (2003) Functional connectivity in the resting brain: A network analysis of the default mode hypothesis. Proc Natl Acad Sci U S A 100:253-258. CrossRef Medline

Hampson M, Driesen N, Roth JK, Gore JC, Constable RT (2010) Functional connectivity between task-positive and task-negative brain areas and its relation to working memory performance. Magn Reson Imaging 28: 1051-1057. CrossRef Medline

Hayama HR, Vilberg KL, Rugg MD (2012) Overlap between the neural correlates of cued recall and source memory: evidence for a generic recollection network? J Cogn Neurosci 24:1127-1137. CrossRef Medline

Jacoby LL (1991) A process dissociation framework: separating automatic from intentional uses of memory. J Mem Lang 30:513-541. CrossRef

Kim H (2010) Dissociating the roles of the default-mode, dorsal, and ventral networks in episodic memory retrieval. Neuroimage 50:1648-1657. CrossRef Medline

Lee SH, Dan Y (2012) Neuromodulation of brain states. Neuron 76:209_ 222. CrossRef Medline

Mandler G (1980) Recognizing: the judgment of previous occurrence. Psychol Rev 87:252-271. CrossRef

Power JD, Petersen SE (2013) Control-related systems in the human brain. Curr Opin Neurobiol 23:223-228. CrossRef Medline

Power JD, Cohen AL, Nelson SM, Wig GS, Barnes KA, Church JA, Vogel AC, Laumann TO, Miezin FM, Schlaggar BL, Petersen SE (2011) Functional network organization of the human brain. Neuron 72:665-678. CrossRef Medline

Ranganath C, Heller A, Cohen MX, Brozinsky CJ, Rissman J (2005) Functional connectivity with the hippocampus during successful memory formation. Hippocampus 15:997-1005. CrossRef Medline

Ranganath C, Heller AS, Wilding EL (2007) Dissociable correlates of two classes of retrieval processing in prefrontal cortex. Neuroimage 35:16631673. CrossRef Medline

Rissman J, Gazzaley A, D’Esposito M (2004) Measuring functional connectivity during distinct stages of a cognitive task. Neuroimage 23:752-763. CrossRef Medline

Rugg MD (2004) Retrieval processing in human memory: electrophysiological and fMRI evidence. In: The cognitive neurosciences, Ed 3, pp 727737. Cambridge, MA: MIT

Rugg MD, Vilberg KL (2013) Brain networks underlying episodic memory retrieval. Curr Opin Neurobiol 23:255-260. CrossRef Medline

Schedlbauer AM, Copara MS, Watrous AJ, Ekstrom AD (2014) Multiple interacting brain areas underlie successful spatiotemporal memory retrieval in humans. Sci Rep 4:6431. CrossRef Medline

Schölvinck ML, Maier A, Ye FQ, Duyn JH, Leopold DA (2010) Neural basis of global resting-state fMRI activity. Proc Natl Acad Sci U S A 107:10238 10243. CrossRef Medline

Seeley WW, Menon V, Schatzberg AF, Keller J, Glover GH, Kenna H, Reiss AL, Greicius MD (2007) Dissociable intrinsic connectivity networks for salience processing and executive control. J Neurosci 27:2349-2356. CrossRef Medline

Smith SM, Miller KL, Salimi-Khorshidi G, Webster M, Beckmann CF, Nichols TE, Ramsey JD, Woolrich MW (2011) Network modelling methods for FMRI. Neuroimage 54:875-891. CrossRef Medline

Spaniol J, Davidson PS, Kim AS, Han H, Moscovitch M, Grady CL (2009) Event-related fMRI studies of episodic encoding and retrieval: metaanalyses using activation likelihood estimation. Neuropsychologia 47: 1765-1779. CrossRef Medline

Staresina BP, Cooper E, Henson RN (2013) Reversible information flow across the medial temporal lobe: the hippocampus links cortical modules during memory retrieval. J Neurosci 33:14184-14192. CrossRef Medline

Tambini A, Ketz N, Davachi L (2010) Enhanced brain correlations during rest are related to memory for recent experiences. Neuron 65:280-290. CrossRef Medline 
Tulving E (1985) Memory and consciousness. Can Psychol Can 26:1-12. CrossRef

Tzourio-Mazoyer N, Landeau B, Papathanassiou D, Crivello F, Etard O, Delcroix N, Mazoyer B, Joliot M (2002) Automated anatomical labeling of activations in SPM using a macroscopic anatomical parcellation of the MNI MRI single-subject brain. Neuroimage 15:273-289. CrossRef Medline

Van Essen DC, Drury HA, Dickson J, Harwell J, Hanlon D, Anderson CH (2001) An integrated software suite for surface-based analyses of cerebral cortex. J Am Med Inform Assoc 8:443-459. CrossRef Medline

Vilberg KL, Rugg MD (2012) The neural correlates of recollection: transient versus sustained FMRI effects. J Neurosci 32:15679-15687. CrossRef Medline

Vilberg KL, Rugg MD (2014) Temporal dissociations within the core recollection network. Cogn Neurosci 5:77-84. CrossRef Medline

Wang L, Laviolette P, O'Keefe K, Putcha D, Bakkour A, Van Dijk KR, Pihlajamäki M, Dickerson BC, Sperling RA (2010) Intrinsic connectivity between the hippocampus and posteromedial cortex predicts memory performance in cognitively intact older individuals. Neuroimage 51:910 917. CrossRef Medline

Watrous AJ, Tandon N, Conner CR, Pieters T, Ekstrom AD (2013) Frequency-specific network connectivity increases underlie accurate spatiotemporal memory retrieval. Nat Neurosci 16:349-356. CrossRef Medline

Wig GS, Grafton ST, Demos KE, Wolford GL, Petersen SE, Kelley WM (2008) Medial temporal lobe BOLD activity at rest predicts individual differences in memory ability in healthy young adults. Proc Natl Acad Sci U S A 105:18555-18560. CrossRef Medline

Wig GS, Schlaggar BL, Petersen SE (2011) Concepts and principles in the analysis of brain networks. Ann N Y Acad Sci 1224:126-146. CrossRef Medline

Yonelinas AP (2002) The nature of recollection and familiarity: a review of 30 years of research. J Mem Lang 46:441-517. CrossRef

Yonelinas AP, Jacoby LL (1995) The relation between remembering and knowing as bases for recognition: effects of size congruency. J Mem Lang 34:622-643. CrossRef 